\title{
Nonlinear Characterization of Safranin O Dye for Application in Optical Limiting
}

\author{
G. Balaji, R.K. Rekha* And A. Ramalingam \\ Centre for Laser Technology, Department of Physics, Anna University, Chennai-600025, India
}

(Received February 21, 2010; in final form May 5, 2010)

\begin{abstract}
The optical nonlinearity of safranin $\mathrm{O}$ dye in methanol solution at different concentrations and as a solid film with polymethylmethacrylate (PMMA) has been studied using cw Nd-YAG laser at $532 \mathrm{~nm}$ as the source of excitation. The optical responses were characterized by measuring the intensity dependent refractive index $\left(n_{2}\right)$ of the medium using the $Z$-scan technique. The open aperture $Z$-scan trace of the dye in solution and solid sample displayed saturable absorption. The closed aperture $Z$-scan trace of the dye exhibited a negative (defocusing) nonlinearity. The nonlinear refractive index was found to vary with concentration. Optical limiting characteristics of the dye at various concentrations in solution were studied. The nonlinearity appears to be of thermal origin.
\end{abstract}

PACS: $42.70 . J \mathrm{k}, 42.65 .-\mathrm{k}, 82.35 .-\mathrm{x}$

\section{Introduction}

Rapid technological advancements in optics have placed great demand on the development of nonlinear optical (NLO) materials with prominent applications in optical limiting and all optical switching [1-3]. Extremely large number of organic compounds with delocalized electron and conjugated double bond systems and a large dipole moment have been synthesized to realize the susceptibilities far larger than the inorganic optical materials [4].

$Z$-scan technique [5,6], based on the spatial distortion of a laser beam, passed through a nonlinear optical material, is widely used in material characterization because of its simplicity, high sensitivity and well-elaborated theory. The opportunity to conduct simultaneous measurements of various nonlinear optical parameters in one set of experiments also makes this technique attractive and applicable for different materials. This method yields both the sign and the magnitude of the nonlinearity, and the value of the nonlinear refractive index, $n_{2}$, may be easily extracted from experimental data with a minimum of analysis [7-9]. $Z$-scan studies also yield important information regarding the response time and the dynamics of the transient processes contributing to the nonlinear refractive index [10]. In the present study, safranin $\mathrm{O}$ from safranin family was chosen, because the dye covers the wavelength region $500-700 \mathrm{~nm}$ and is generally very efficient.

Optical limiting is a nonlinear optical process in which the transmitted intensity of a material decreases with increased incident light intensity. Optical limiting performance will be enhanced by coupling two or more of

\footnotetext{
* corresponding author; e-mail: pouchasri@yahoo.com
}

the nonlinear optical mechanisms. Excited state absorption (ESA) and reverse saturable absorption (RSA) are the most common mechanisms for the nonlinear optical behavior of organic materials [11]. Nonlinear optical effects can be employed for the design and performance of optical limiter [12-14]. It has been demonstrated that optical limiting can be used for the protection of eyes and sensors from intense lasers [15]. In this paper, the optical nonlinearity and optical limiting action of dye in methanol solvent and thin film using PMMA at $50 \mathrm{~mW}$ cw Nd-YAG laser power at the wavelength of $532 \mathrm{~nm}$ was studied. The experiment was repeated for different dye concentration and the third order nonlinear refractive index was found to be linearly dependent on the dye concentration within the range studied.

\section{Experiment}

\subsection{Materials}

The safranin O dye obtained from Loba Chemie, India, was chosen for the study. The chemical structures of the dye are shown in Fig. 1. Thin layer chromatography (TLC) test confirms the absence of any impurities in the dye. Methylmethacrylate (MMA) (Lancaster) was chosen as a monomer for synthesizing dye doped polymer film. Spectroscopic grade methanol was chosen as additive, because it has good solubility for the dye and enhances the laser damage threshold. Benzoyl peroxide was used as the initiator.

\subsection{Synthesis of dye doped polymer films}

MMA is used as the monomer for the preparation of the dye doped polymer (DDP) film. MMA and methanol are taken in the ratio $4: 1(\mathrm{v} / \mathrm{v})$. Known weight of the dye is dissolved in this mixture. $0.5 \mathrm{~g}$ of benzoyl peroxide, per 
<smiles>Cc1cc2nc3cc(C)c(N)cc3[n+](-c3ccccc3)c2cc1N</smiles>

Fig. 1. Chemical structure and molecular formula of safranin $\mathrm{O}$ dye $\mathrm{C}_{20} \mathrm{H}_{19} \mathrm{~N}_{4} \mathrm{Cl}$.

$100 \mathrm{ml}$ of MMA solution is used as an initiator for polymerization. The solution taken in polymerizing tubes is deoxygenated in a nitrogen stream, and the glass containers are sealed. Thermal bulk free-radical polymerization [16-18] is carried out in a temperature controlled water bath and the temperature is maintained at $35^{\circ} \mathrm{C}$ for 2 days and at $40^{\circ} \mathrm{C}$ for another 2 days till the solution becomes viscous. The DDP film of desired concentration was synthesized by pouring the viscous dye solution with initiator mixture onto a Petri dish placed in a glass enclosure to prevent from dust, during natural drying. The optical quality of this film is checked by passing $\mathrm{He}-\mathrm{Ne}$ laser beam of power $5 \mathrm{~mW}$. The film which shows no distortion or dispersion of the laser beam alone is taken for further studies. The DDP film of concentration $0.05 \mathrm{mM}$ and thickness of $0.95 \mathrm{~mm}$ was synthesized.

\subsection{Absorption spectra}

The UV-Vis absorption spectrum of the dye in methanol solvent was obtained using a Perkin-Elmer Lambda 35 spectrophotometer and is shown in Fig. 2. The spectral parameters such as absorption-peak wavelength $(\lambda)$, molar-extinction coefficient $(\varepsilon)$, oscillator strength $(f)$, band width $\left(\Delta \nu_{1 / 2}\right)$, were calculated to be $512 \mathrm{~nm}, 1.01 \times 10^{5} \mathrm{~L} \mathrm{~mol}^{-1} \mathrm{~cm}^{-1}, 1.53 \times$ $10^{-24} \mathrm{~L} \mathrm{~mol}^{-1} \mathrm{~cm}^{-2}$ and $3.52 \times 10^{3} \mathrm{~cm}^{-1}$, respectively.

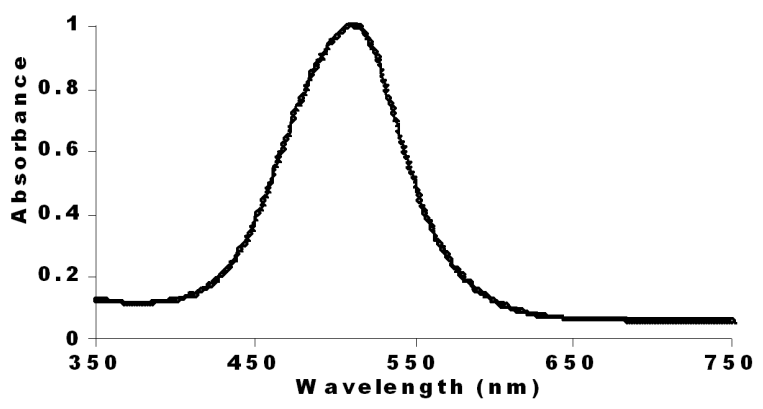

Fig. 2. UV-Vis absorption spectra of the safranin $\mathrm{O}$ dye in methanol.

\subsubsection{Z-scan technique for determining the nonlinear} refractive index

A diode-pumped Nd:YAG laser of wavelength $532 \mathrm{~nm}$ (Coherent Compass TM 215M-50) is used as the excita- tion source for the $Z$-scan technique. The laser of Gaussian beam profile was focused by a convex lens, of focal length, $f=3.5 \mathrm{~cm}$ to produce a beam waist $\omega_{0}$ of $20 \mu \mathrm{m}$. The peak intensity of the incident laser beam is $I_{0}=3.5 \mathrm{~kW} / \mathrm{cm}^{2}$. The diffraction length, $Z_{\mathrm{R}}$ was found to be $2.52 \mathrm{~mm}$. The schematic of the experimental setup used is shown in Fig. 3. A $1 \mathrm{~mm}$ wide optical cell containing the dye in solvent is translated across the focal region along the axial direction that is the direction of the propagation laser beam. The transmission of the beam through an aperture placed in the far field is measured using photodetector fed to the digital power meter (Field master Gs-coherent). For an open aperture $Z$-scan, a lens to collect the entire laser beam transmitted through the sample replaced the aperture. The same was repeated for the polymer film.

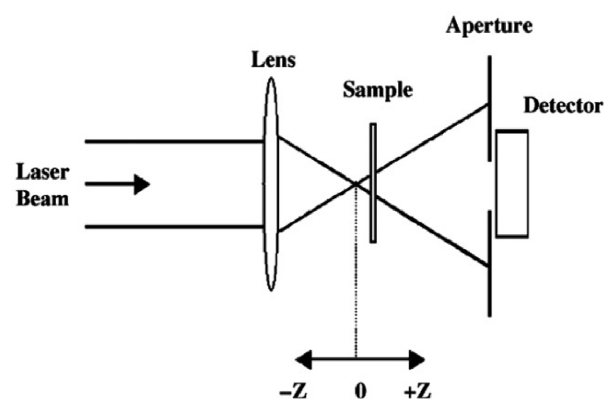

Fig. 3. Experimental setup for $Z$-scan.

\subsubsection{Optical limiting technique}

The limiting effect of the dye was studied by using a $50 \mathrm{~mW}$ Nd-YAG cw laser at $532 \mathrm{~nm}$. The experimental setup for the demonstration of optical limiting is shown in Fig. 4. A $1 \mathrm{~mm}$ quartz cuvette containing nonlinear material (sample solution) is kept at the position where the transmitted intensity shows a valley in closed aperture $Z$-scan curve $[18,19]$. A variable beam splitter was used to vary the input power. An aperture $A$ of variable diameter is used to control the cross-section of the beam coming out of the sample cuvette. This beam is then made to fall on the photodetector (PD). The input laser intensity is varied systematically and the corresponding output intensity values were measured by the photodetector. At very high peak intensities (closer to the focus) we could observe diffraction type pattern with concentric ring structures probably due to self-phase modulation. However, in limiting experiments we have ensured that there is no ring pattern formation by placing the sample away from focus.

\section{Results and discussion}

The safranin $\mathrm{O}$ dye in methanol and polymer film at various concentrations for the incident intensity $I_{0}=$ $3.5 \mathrm{~kW} / \mathrm{cm}^{2}$ was evaluated by the measurements of $Z$-scan. The saturation absorption for the dye in solvent and polymer film is shown by the open $Z$-scan curve in 


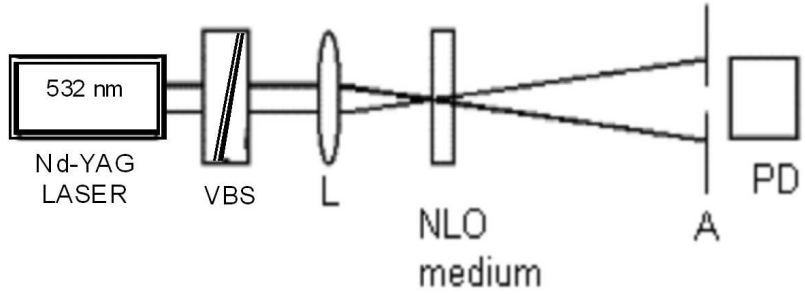

Fig. 4. Experimental setup for measuring the limiting effect.

Fig. 5. The transmission at the focus decreases with increasing sample concentration. At higher concentration, the sample gives better nonlinear optical properties. Absorption saturation in the sample enhances the peak and decreases the valley in the closed aperture $Z$-scan and results in distortions in the symmetry of the $Z$-scan about $Z=0$. The peak followed by a valley-normalized transmittance curve obtained from the closed aperture $Z$-scan data, indicates that the sign of the refraction nonlinearity is negative, i.e. self-defocusing. The self-defocusing effect is due to the local variation of the refractive index with temperature. The defocusing effect for the dye in solvent and polymer film shown in Fig. 6, is attributed to a thermal nonlinearity resulting from the absorption of radiation at $532 \mathrm{~nm}$. The measurable quantity $\Delta T_{\mathrm{p}-\mathrm{v}}$ can be defined as the difference between the normalized peak and valley transmittances. The pure nonlinear refractive index $n_{2}$ was obtained by dividing the closed aperture data by the open aperture data [6]. The pure nonlinear refraction $Z$-scan curves are shown in Fig. 7 for the dye in solvent and polymer film. Experimentally determined nonlinear refractive index $n_{2}$ and nonlinear absorption coefficient $\beta$ can be used in finding the absolute value of the third-order nonlinear optical susceptibility. In order to know the contribution from the solvent to the observed nonlinear response, the $Z$-scan was performed on pure solvent. Neither nonlinear absorption nor nonlinear refraction was observed. The value of $\Delta T_{\mathrm{p}-\mathrm{v}}$ has increased for the dye doped polymer film when compared to the dye in methanol. This may be due to the heat dissipation being faster in liquids as compared to that in a solid medium. The experimentally determined values of $\Delta T_{\mathrm{p}-\mathrm{v}}, n_{2}, \beta, \Delta n\left(\Delta n=n_{2} I_{0}\right)$ and $\chi^{3}$ are given in Table I.

Nonlinear parameters of safranin $\mathrm{O}$ dye in solvent and film.

TABLE I

\begin{tabular}{c|c|c|c|c|c|c}
\hline \hline \multicolumn{7}{c}{ Nonlinear parameters of safranin O dye in methanol } \\
\hline \multirow{2}{*}{ Medium } & Concentration & $\Delta T_{\mathrm{p}-\mathrm{v}}$ & $\begin{array}{c}n_{2} \times 10^{-7} \\
{\left[\mathrm{~cm}^{2} / \mathrm{W}\right]}\end{array}$ & $\begin{array}{c}\beta \times 10^{-3} \\
{[\mathrm{~cm} / \mathrm{W}]}\end{array}$ & $\begin{array}{c}\Delta n=n_{2} I_{0} \\
\times 10^{-4}\end{array}$ & $\begin{array}{c}\left|\chi^{(3)}\right| \times 10^{-6} \\
{[\text { e.s.u. }]}\end{array}$ \\
\hline \multirow{3}{*}{ solvent } & $0.02 \mathrm{mM}$ & 0.59 & -0.40 & -0.36 & -1.33 & 1.80 \\
& $0.03 \mathrm{mM}$ & 0.99 & -0.78 & -0.40 & -2.60 & 3.51 \\
& $0.04 \mathrm{mM}$ & 1.47 & -1.16 & -0.43 & -3.87 & 5.18 \\
& $0.05 \mathrm{mM}$ & 1.84 & -1.45 & -0.47 & -4.86 & 6.54 \\
\hline polymer film & $0.05 \mathrm{mM}$ & 2.18 & -1.72 & -0.52 & -5.76 & 7.76
\end{tabular}

From Fig. 8 (a and b), there is an increasing trend for the values of $n_{2}$ and $\beta$ as the concentration increases. This may be attributed to the fact that the number of dye molecules increases as the concentration increases, more particles are thermally agitated resulting in an enhanced effect.

The nonlinear refractive index of organic dye solution is due to thermal heating. An important parameter which decides on the magnitude of such thermal effects is the rate of change of refractive index with temperature $(\mathrm{d} n / \mathrm{d} T)$ for the dye solvent [10]. The organic dye in solvent will have a tendency to form non-fluorescent dimers. With increasing dye concentration there is an increase in dimer concentration. These dimers have shorter wavelength than monomers and are weakly fluorescent. Such a dimer is expected to absorb light. Thus for the inci- dent radiation, the fraction absorbed by non-fluorescent dimers, eventually raise the temperature of the medium.

The values of $n_{2}$ in dye doped polymer film were found to have larger values than in the case of dye in solvent. This may be due to the Anderson localization of photons [20].

The scattering mean free path of photons in solids is smaller than in the case of liquids, so the localization of strong electromagnetic field inside the solid is responsible for the increase in nonlinearity in films. The UV-Vis absorption spectra of the samples recorded before and after the laser irradiation show that the pattern and intensity of the spectra have no change. This indicates that all the dye samples possess good photostability. The source used to probe the nonlinear material is a continuous wave laser; the optical nonlinearity of the dye, ob- 


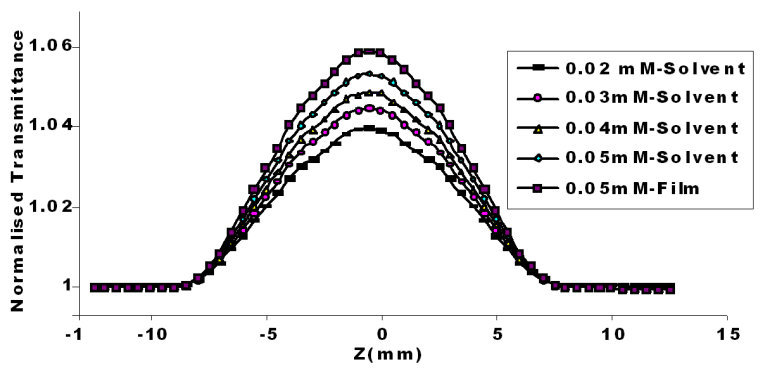

Fig. 5. Open $Z$-scan curve - safranin O dye in solvent and film.

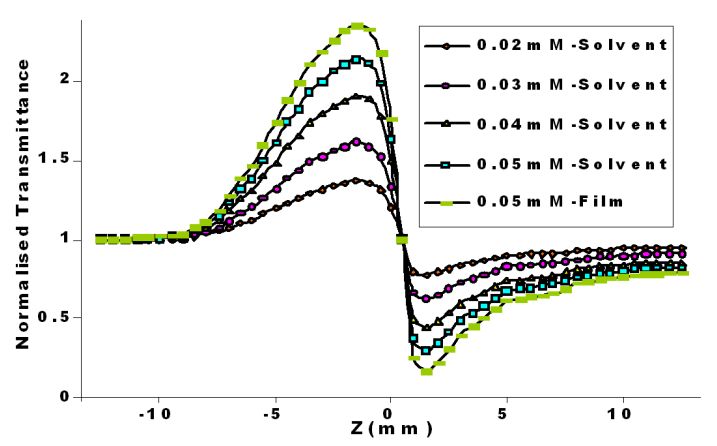

Fig. 6. Closed $Z$-scan curve - Safranin O dye in solvent and film.

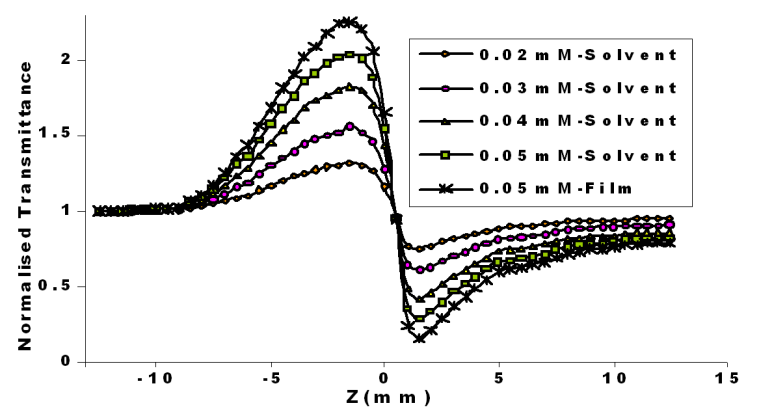

Fig. 7. Pure nonlinear refraction curve - safranin $\mathrm{O}$ dye in solvent and film.
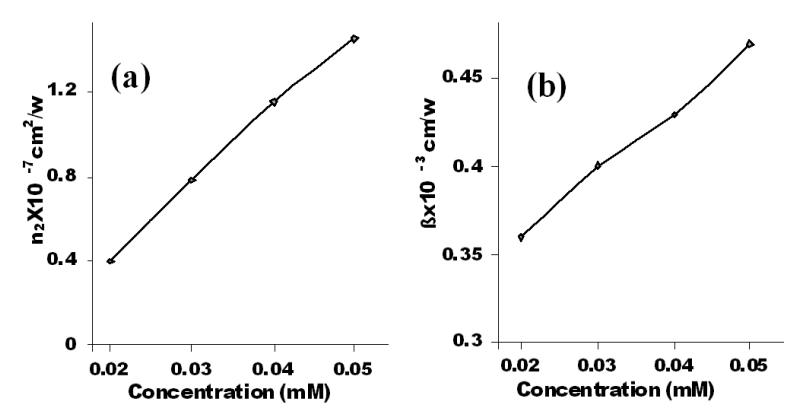

Fig. 8. Concentration dependence (a) $n_{2}$, (b) $\beta$ of safranin $\mathrm{O}$ dye in solvent. served here, is likely to be of thermal origin arising from the temperature dependence of refractive index of sample which acts as a lens. The phase of the propagating beam will be distorted due to the presence of this thermal lens. The peak-valley separation of more than 1.7 times of Rayleigh range also suggests the presence of thermal component [21]. It is worth noting that the value of $\chi^{3}$ for the dye studied is larger than those of some representative third-order nonlinear optical materials such as chalcone and its derivatives and organic dyes like croconium $[22,23]$.

The optical limiting curves obtained with a $50 \mathrm{~mW}$ Nd:YAG laser of wavelength $532 \mathrm{~nm}$ for the dye in solution at different concentrations are shown in Fig. 9. The output power rises initially with increase in input power, but after a certain threshold value the samples start defocusing the beam, resulting in a greater part of the beam cross-section being cut off by the aperture. Thus the transmittance recorded by the photodetector remained reasonably constant showing a plateau region. At incident power above $20 \mathrm{~mW}$, the output power tends to be constant, because its nonlinear absorption coefficient increases with increase in the incident irradiance. In liquids, where the thermal expansion is large, high absorbance of the nonlinear material at the corresponding wavelength leads to increase in temperature and density of the sample. Heating due to laser absorption is responsible for changing the absorption coefficient and optical limiting effect [24].

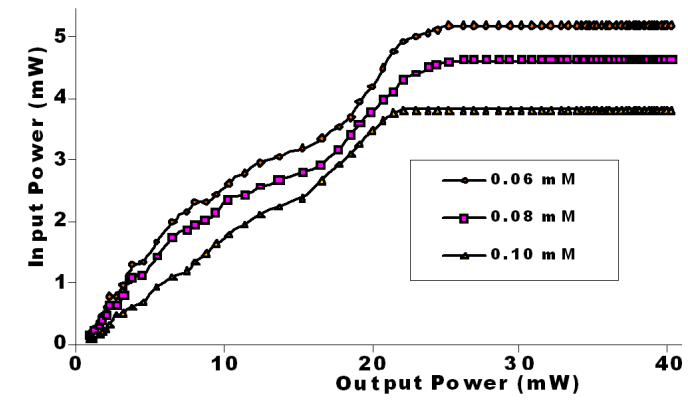

Fig. 9. Optical limiting curves of safranin $\mathrm{O}$ dye in solvent.

Separate optical limiting experiment was performed on pure methanol and it is found to have no contribution to optical limiting in the power range of the laser used. The dye investigated here is very weakly fluorescent and is nonfluorescent at the wavelength studied, optimizing the conversion of the absorption energy into heat [24]. The optical limiting effect shows an increase with increasing the concentration of the dye solution as shown in Fig. 10. The optical limiting responses of the low concentration solutions are generally much weaker than those of high concentrated solutions, while high concentrated solution exhibits strong optical limiting. This indicates that the number density of dye molecules in the laser beam is the main factor affecting the clamped level. From the 


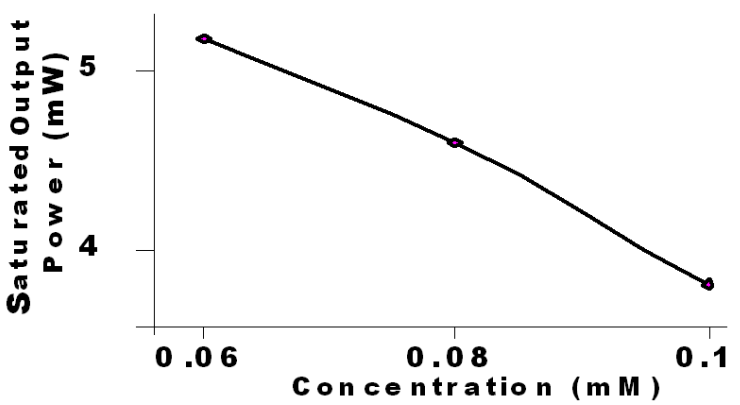

Fig. 10. Concentration dependence of saturated output power of safranin $\mathrm{O}$ dye in solvent.

threshold intensity for optical limiting for each sample, it can be seen that the optical power limiting threshold is inversely proportional to the concentration. The data show that as the concentration increases, a reduction in linear transmittance as well as the clamping level is observed. The experimentally determined optical limiting saturated output power values are shown in Table II. The results were comparable to some of the reports of low power optical limiting [25]. Therefore the sample possesses limiting effect for the light of $532 \mathrm{~nm}$.

\section{TABLE II}

Concentration dependence of saturated output power of safranin $\mathrm{O}$ dye.

\begin{tabular}{c|c}
\hline \hline Concentration $[\mathrm{mM}]$ & Saturated output power $[\mathrm{mW}]$ \\
\hline 0.06 & 5.18 \\
0.08 & 4.59 \\
0.10 & 3.80
\end{tabular}

\section{Conclusion}

The third order nonlinear optical properties and optical limiting behavior of safranin $\mathrm{O}$ dye have been studied. Both NLA and NLR contribute to the large third-order nonlinearity of the dye. The origin of optical nonlinearity observed in the cw regime is attributed to the thermal variation of refractive index in the medium. The aperture limited designs based on thermo-optic nonlinearity such as the one studied here can be used as efficient limiters in the $\mathrm{cw}$ regime.

\section{Acknowledgments}

The authors wish to thank the DAE-BRNS for their financial support.

\section{References}

[1] N. Venkatram, D. Narayana Rao, L. Giribabu, S. Venugopal Rao, Chem. Phys. Lett. 464, 211 (2008).
[2] Ting Huang, Zhonghua Hao, Hongmei Gong, Zijun Liu, S. Xiao, S. Li, Y. Zhai, S. You, Q. Wang, J. Qin, Chem. Phys. Lett. 451, 213 (2008).

[3] M. George, C.I. Muneer, C.P. Singh, K.S. Bindra, S.M. Oak, Opt. Laser Technol. 40, 373 (2008).

[4] Tapati Mallik, Tanusree Kar, J. Cryst. Growth 285, 178 (2005).

[5] M. Sheik-Bahae, A.A. Said, E.W. Van Stryland, Opt. Lett. 14, 955 (1989).

[6] M. Sheik-Bahae, A.A. Said, T.H. Wei, D.J. Hagan, E.W. Van Stryland, IEEE J. Quant. Electron. 26, 760 (1990).

[7] O.V. Przhonska, J.H. Lim, D.J. Hagan, E.W. Van Stryland, J. Opt. Soc. Am. B 15, 802 (1998).

[8] J. Wang, M. Sheik-Bahae, A.A. Said, D.J. Hagan, E.W. Van Stryland, J. Opt. Soc. Am. B 11, 1009 (1994).

[9] T. Geethakrishnan, P.K. Palanisamy, Opt. Commun. 270, 424 (2007).

[10] S. Sinha, A. Ray, K. Dasgupta, J. Appl. Phys. 87, $3222(2000)$.

[11] K. Jamshidi-Ghaleh, S. Salmani, M.H. Majles Ara, Opt. Commun. 271, 551 (2007).

[12] S. Qi, C. Zhang, X. Yang, K. Chen, L. Zhang, Y. Liu, T. Xu, J. Tian, Optik 118, 425 (2007).

[13] S. Qi, C. Zhang, X. Yang, K. Chen, L. Zhang, G. Yang, X. Liang, T. Xu, J. Tian, Opt. Mater. 29, 1348 (2007).

[14] B. Valasalmilka, G. Sreekumar, C.I. Muneera, Kaladevi Sendhil, C. Vijayan, J. Mater. Sci. 40, 777 (2005).

[15] Q.S. Li, C.L. Liu, L.Y. Zang, Q.H. Gong, X.L. Yu, C.B. Cao, Laser Phys., 18, 434 (2008).

[16] A. Costela, I. Garcia Marino, J.M. Figuera, F. Amat-Guerri, J. Barroso, R. Sastre, Opt. Commun. 130, 44 (1996).

[17] V. Sindhu Sukumaran, A. Ramalingam, Spectrochim. Acta Part A 63, 673 (2006).

[18] R.K. Rekha, A. Ramalingam, J. Mod. Opt. 56, 1096 (2009).

[19] K. Sendhil, C. Vijayan, M.P. Kothiyal, Opt. Laser Technol. 38, 512 (2006).

[20] S. Sharma, D. Mohan, S.K. Ghoshal, Opt. Commun. 281, 2923 (2008).

[21] S.J. Mathews, S. Chaitanya Kumar, L. Giribabu, S. Venugopal Rao, Mater. Lett. 61, 4426 (2007).

[22] H.J. Ravindra, A. JohnKiran, K. Chandrasekaran, H.D. Shashikala, S.M. Dharmaprakash, Appl. Phys. B (Laser Optics) 88, 105 (2007).

[23] Z. Li, Z.-H. Jin, K. Kasatani, H. Okamoto, S. Takenaka, J. Appl. Phys. 44, 4956 (2005).

[24] M. Rashidian, D. Dorranian, S. Ahmadi Darani, S. Saghafi, M. Ghoranneviss, Optik, 2008, in press, DOI:10.1016/j.ijleo.2008.05.001.

[25] S. Kaladevi, C. Vijayan, M.P. Kothiyal, Opt. Mater. 27, 1606 (2004). 